\title{
Non-split and Inverse Non-split Domination Numbers in the Join and Corona of Graphs
}

\author{
Esamel M. Paluga \\ Department of Mathematics \\ Caraga State University \\ Ampayon, Butuan City, Philippines
}

\author{
Rolando N. Paluga \\ Department of Mathematics \\ Caraga State University \\ Ampayon, Butuan City, Philippines
}

\begin{abstract}
A dominating set $D$ of a graph $G=(V, E)$ is non-split dominating set if $\langle V \backslash D\rangle$ is connected. The non-split domination number of $G$ is the minimum cardinality of a non-split dominating set in $G$. Let $D$ be a minimum dominating set in $G$. If a subset $D^{\prime}$ of $V \backslash D$ is dominating in $G$, then $D^{\prime}$ is called an inverse dominating set with respect to $D$. Furthermore, if $\left\langle V \backslash D^{\prime}\right\rangle$ is connected, then $D^{\prime}$ is called an inverse non-split dominating set. The inverse non-split domination number of $G$ is the minimum cardinality of an inverse non-split dominating set in $G$. In this paper, characterization of non-split dominating sets in the join and corona of two graphs are presented. Furthermore, explicit formulas for determining the non-split and inverse nonsplit domination numbers of these graphs are also determined.
\end{abstract}

\section{General Terms:}

Graph Theory, Domination

\section{Keywords:}

non-split domination, inverse non-split domination, join, coronaifx

\section{INTRODUCTION}

Given a connected graph $G=(V(G), E(G))$ and $D \subseteq V(G)$, we say $D$ is dominating set in $G$ if for all $x \in V(G) \backslash D$, there exists $y \in D$ such that $d_{G}(x, y)=1$. The domination number of $G$, denoted by $\gamma(G)$, is the minimum cardinality of all dominating sets in $G$. If $D$ is dominating and $\langle V(G) \backslash D\rangle$ is connected, then we say that $D$ is non-split dominating set (or ns-dominating)in $G$. The non-split dominating number of $G$, denoted by $\gamma_{n s}(G)$, is the minimum cardinality of all non-split dominating sets in $G$. Let $D$ be a minimum dominating set in $G$. If there exists $D^{\prime} \subseteq V(G) \backslash D$ such that $D^{\prime}$ is non-split dominating set, then we say that $D^{\prime}$ is an inverse non-split dominating set (or ins-dominating) in $G$. The minimum cardinality of all inverse non-split dominating sets in $G$ is the inverse nonsplit domination number of $G$ and is denoted by $\gamma_{n s}^{\prime}(G)$. To illustrate these concepts, consider the following examples:

EXAMPLE 1.1. Consider the graph in Figure 1. Let $D=\{a, d$,$\} , then \langle V(G) \backslash D\rangle$ is connected. Note that $D$ is minimum. Hence, $\gamma_{n s}(G)=2$. Moreover, if $D^{\prime}=\{c, f\}$, then $D^{\prime} \subseteq V(G) \backslash D$ and $\left\langle V(G) \backslash D^{\prime}\right\rangle$ is connected. Since $D^{\prime}$ is minimum, it follows that $\gamma_{n s}^{\prime}(G)=2$.

The join of any graphs $G$ and $H$, denoted by $G+H$, is the graph with $V(G+H)=V(G) \cup V(H)$ and $E(G+H)=$ $E(G) \cup E(H) \cup\{u v: u \in V(G)$ and $v \in V(H)\}$. The corona

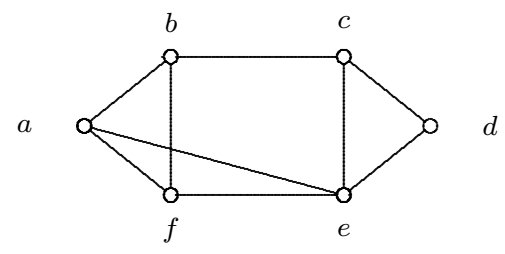

Figure 1: A graph $G$ with $\gamma_{n s}(G)=2$ and $\gamma_{n s}^{\prime}(G)=2$

of $G$ and $H$, denoted by $G \circ H$ is defined as the graph obtained by taking one copy of $G$ (which has $p$ vertices) and $p$ copies of $H$, and then joining the ith vertex of $G$ to every vertex in the ith copy of $H$. We denote the copy of $H$ with respect to vertex $a \in V(G)$ by $H^{a}$. A path with vertices $x_{0}, x_{1}, \ldots, x_{n}$ will be denoted by $\left[x_{0}, x_{1}, \ldots, x_{n}\right]$. The distance $d_{G}=(u, v)$ between vertices $u$ and $v$ is the lenght of a shortest path connecting $u$ and $v$. An extreme vertex in $G$ is a vertex that is adjacent to all other vertices of $G$. The set $\operatorname{ext}(G)$ consists of all the extreme vertices of $G$.

Throughout this paper, $G=(V(G), E(G))$ is a simple undirected and connected graph. The order of $G$ denoted by $|G|$, is the cardinality of $V(G)$. For other graph theoretic terms which are assumed here, readers are advised to refer to [5]. The following are needed in the development of this paper.

\section{NON-SPLIT DOMINATION IN THE JOIN AND CORONA OF GRAPHS}

This section characterizes all non-split dominating sets in the join and corona of two graphs $G$ and $H$. As a consequence, the explicit formulas for the non-split dominating numbers of these graphs are presented. The following results are necessary in the development of succeding results

REMARK 2.1. For any connected graph $G, \gamma_{n s}(G) \geq 1$.

LEMMA 2.2. [7] Let $G$ be a connected graph. Then

$$
\gamma(G) \leq \frac{|G|}{2}
$$

THEOREM 2.3. Let $G$ and $H$ be connected graphs. Then $D \subseteq V(G+H)$ is ns-dominating in $G+H$ if and only one of the following conditions holds:

(i) $D \cap V(G) \neq \emptyset, V(G)$ and $D \cap V(H) \neq \emptyset, V(H)$;

(ii) $D$ is dominating in $H$;

(iii) $D$ is dominating in $G$;

(iv) $V(G) \subseteq D$, and $\langle V(H) \backslash D\rangle$ is connected;

(v) $V(H) \subseteq D$, and $\langle V(G) \backslash D\rangle$ is connected. 
Proof. If $V(G) \cap D \neq \emptyset, V(G)$ and $V(H) \cap D \neq \emptyset, V(H)$, then we are done. Suppose $V(G) \cap D=\emptyset$. Then $D \subseteq V(H)$. Let $x \in V(H) \backslash D$. Then $x \in V(G+H) \backslash D$. Since $D$ is dominating in $G+H, \exists y \in D$ such that $d_{G+H}(x, y)=1$. Since $V(G) \cap D=\emptyset, y \in V(H)$. This implies that $y \in V(H) \cap D$. Consequently, $V(H) \cap D$ is dominating in $H$. Thus, $(i i)$ holds. The case $($ iii $)$ is proved similarly.

Suppose $V(G) \cap D=V(G)$. If $V(H) \cap D=\emptyset$, then $\langle V(H) \backslash D\rangle=H$ is connected. Suppose $V(H) \cap D \neq \emptyset$. Let $x, y, \in V(H) \backslash D$. Since $V(H) \backslash D=V(G+H) \backslash D$, and $\langle V(G+H) \backslash D\rangle$ is connected, $\langle V(H) \backslash D\rangle$ is connected, proving $(i v)$. The case $(v)$ is proved similarly.

Suppose that $D \cap V G)=\emptyset$ and $x \in V(H) \backslash D \subseteq V(G+$ $H) \backslash D$. Since $D$ is dominating in $G+H, \exists y \in D$ such that $d_{G+H}(x, y)=1$. Now, $D \cap V(G)=\emptyset$ so $y \in V(H) \cap D$ and $d_{H}(x, y)=1$. Thus, $V(H) \cap D$ is dominating in $H$. The proof for case $(\mathrm{v})$ is similarly done.

For the converse, assume $(i)$ holds. Let $x \in V(G+H) \backslash D$. Let $y \in D \cap V(G), z \in D \cap V(H), q \in V(G) \backslash D$ and $r \in V(H) \backslash D$. Either $x \in V(G) \backslash D$ or $x \in V(H) \backslash D$. If $x \in V(G) \backslash D$, then $d_{G+H}(x, z)=1$. If $x \in V(H) \backslash D$, then $d_{G+H}(w, y)=$ 1. Hence, $D$ is dominating in $G+H$. Furthermore, let $u, v \in$ $V(G+H) \backslash D$. If $u, v, \in V(G) \backslash D$, then $[u, r, v]$ is a path with vertices in $V(G+H) \backslash D$. If $u, v, \in V(H) \backslash D$, then $[u, q, v]$ is a path $G+H$ connecting $u$ and $v$. Lastly, if $u \in V(G) \backslash D$ and $v \in$ $V(H) \backslash D$ then $[x, y]$ is a path in $G+H$. Thus, $\langle V(G+H) \backslash D\rangle$ is connected. Hence, $D$ is ns-dominating in $G+H$.

Next, we assume that $(i i)$ holds. Let $x \in V(G+H) \backslash D$. Since $D \cap V(G)=V(G), V(G+H) \backslash D=V(H) \backslash D$. Consequently, $d_{G+H}(x, y)=1$ where $y \in D \cap V(G)$. This implies that $D$ is dominating in $G+H$. Furthermore, since $\langle V(H) \backslash D\rangle$ is connected, $\langle V(G+H) \backslash D\rangle$ is connected. Accordingly, $D$ is ns-dominating in $G+H$.

To prove that the result holds assuming $(i i i)$ is similar to $(i i)$.

Netx, assume $i v$ holds. Let $x \in V(G+H) \backslash D$. Then either $x \in V(G)$ or $x \in V(H) \backslash D$. If $x \in V(G)$, then for all $y \in$ $V(H) \backslash D, d_{G+H}(x, y)=1$. If $x \in V(H) \backslash D$, then since $V(H) \cap D$ is dominating in $H, \exists w \in V(H) \cap D$ such that $d_{H}(x, y)=1$. It follows that $\exists w \in D$ such that $d_{G+H}(x, y)=$ 1 . Thus, $D$ is dominating in $G+H$. Moreover, let $u, v \in V(G+$ $H) \backslash D$. If $u, v \in V(G)$, then there exists a path in $G$ connecting $u$ and $v$ since $G$ is connected. If $u, v \in V(H) \backslash D$, then for each $w \in V(G),[u, w, v]$ is a path in $G+H$ connecting $u$ and $v$. If $u \in V(G)$ and $v \in V(H) \backslash D$, then $[u, v]$ is a path in $G+$ $H$ connecting $u$ and $v$. Thus, $\langle V(G+H) \backslash D\rangle$ is connected. Hence, $D$ is ns-dominating in $G+H$.

Lastly, assume that $(v)$ holds. The proof is similar to $(i v)$.

\section{COROLlaRY 2.4. Let $G$ and $H$ be connected graphs. Then}

$$
\gamma_{n s}(G+H)=\left\{\begin{array}{l}
1 \text { if either } \gamma(G)=1 \text { or } \gamma(H)=1, \\
2 \text { otherwise. }
\end{array}\right.
$$

Proof. Suppose $\gamma(G)=1$. Then $\exists x \in V(G)$ such that $D=\{x\}$ is dominating in $G$. By Theorem 2.3 $(i v), D$ is nsdominating in $G+H$. Thus, $\gamma_{n s}(G+H) \leq 1$. Combining this with Remark 1.1, $\gamma_{n s}(G+H)=1$. The case where $\gamma(H)=1$ can be shown similarly using Theorem 2.3 $(v)$.

Now suppose $\gamma(G) \geq 2$ and $\gamma(H) \geq 2$. Let $D=\{a, b\}$ such that $a \in V(G)$ and $b \in V(H)$. Then $D \cap V(G) \neq \emptyset, V(G)$ and $D \cap V(H) \neq \emptyset, V(H)$. By Theorem $2.3(i), D$ is ns-dominating in $G+H$. Thus, $\gamma_{n s}(G+H) \leq 2$. Suppose $\gamma_{n s}(G+H)=1$. Then $\exists D^{\prime} \subseteq V(G+H)$ such that $D^{\prime}=\{y\}$ for some $y \in$ $V(G+H)$. If $y \in V(G)$, then $D^{\prime}$ must be dominating in $G$, contradicting the fact that $\gamma(G) \geq 2$. A similar argument can be used to show that $y \in V(H)$ cannot hold. Thus, $\left|D^{\prime}\right| \geq 2$. Accordingly, $\gamma_{n s}(G+H)=2$.
THEOREM 2.5. Let $G$ and $H$ be connected graphs. Then $D \subseteq V(G \circ H)$ is ns-dominating in $G \circ H$ if and only one of the following conditions holds:

(i) There exists $v \in V(G)$ such that $\langle V(G \circ H) \backslash D\rangle$ is a connected subgraph of $H^{v}$;

(ii) For each $v \in V(G), V\left(H^{v}\right) \cap D$ is dominating in $H^{v}$ and $D \subseteq \cup_{w \in V(G)} V\left(H^{w}\right)$;

(iii) $V(G) \cap D$ is ns-dominating in $G$ and $V\left(H^{v}\right) \subseteq D$ whenever $v \in V(G) \cap D$ and $V\left(H^{v}\right) \cap D$ is dominating in $H^{v}$ whenever $v \in V(G) \backslash D$.

Proof. Assume that $D$ is ns-dominating in $G \circ H$. Suppose $V(G) \cap D=V(G)$, i.e., $V(G) \subseteq D$. If $V(G \circ H) \backslash D=\emptyset$, then $(i)$ follows vacuously. Suppose $V(G \circ H) \backslash D \neq \emptyset$. Since $D$ is ns-dominating in $G \circ H,\langle V(G \circ H) \backslash D\rangle$ is connected. Since $V(G) \subseteq D, V(G \circ H) \backslash D \subseteq \cup_{a \in V(G)} V\left(H^{a}\right)$. Thus, $V(G \circ H) \backslash$ $D \subseteq V\left(H^{v}\right)$ for some $v \in V(G)$. That is, $\langle V(G \circ H) \backslash D\rangle \leq$ $H^{v}$. Hence, $(i)$ holds.

Suppose $V(G) \cap D=\emptyset$. Let $v \in V(G), B=V\left(H^{v}\right) \cap D$, and $x \in V\left(H^{v}\right) \backslash B$. It follows that $x \in V(G \circ H) \backslash D$. Since $D$ is dominating in $G \circ H, \exists y \in D$ such that $d_{G \circ H}(x, y)=1$. Since $x \in V\left(H^{v}\right)$ and $d_{G \circ H}(x, y)=1, y \in V\left(H^{v}\right)$ or $y=v$. If $y=v$, then $y \in V(G) \cap D$, a contradiction. Thus, $y \in V\left(H^{v}\right) \cap$ $D=B$. Accordingly, $B=V\left(H^{v}\right) \cap D$ is dominating in $H^{v}$. Since $V(G) \cap D=\emptyset, D \subseteq \cup_{v \in V(G)} V\left(H^{v}\right)$. Hence, $($ ii $)$ holds. Suppose $V(G) \cap D \neq \emptyset$ and $V(G) \cap D \neq V(G)$. Let $A=$ $V(G) \cap D$ and $x \in V(G) \backslash A$, i.e., $x \in V(G) \backslash D$. It follows that $x \in V(G \circ H) \backslash D$. Since $D$ is dominating in $G \circ H, \exists y \in D$ such that $d_{G \circ H}(x, y)=1$. Suppose $y \notin V(G)$. Then $\exists v \in V(G)$ such that $y \in V\left(H^{v}\right)$. Since $v$ is a cutvertex, $d_{G \circ H}(x, y)=$ $d_{G \circ H}(x, v)+d_{G \circ H}(v, y) \geq 1+1=2$. This is a contradiction. Consequently, $y \in V(G)$. Note that $d_{G}(x, y)=1$. Hence, $A$ is dominating in $G$.

Let $x, y \in V(G) \backslash A=V(G) \backslash D$. Since $\langle V(G \circ H) \backslash D\rangle$ is connected and $x, y \in V(G \circ H) \backslash D$, there exists a geodesic path $P=\left[u_{1}, u_{2}, \ldots, u_{n}\right]$ where $u_{1}=x$ and $u_{n}=y$. Suppose $\exists i$ with $1<i<n$ such that $u_{i} \notin V(G)$. Then $\exists w \in V(G)$ such that $u_{i} \in V\left(H^{w}\right)$. Since $P$ is geodesic, $u_{i-1}=w$. Note that $u_{n} \notin V\left(H^{w}\right)$. Now, $w$ is the only vertex that can be used to traverse from $u_{i}$ going to $u_{n}$. This contradicts the fact that $P$ is a path. Thus $u_{i} \in V(G), \forall i$. Accordingly, $\langle V(G) \backslash A\rangle$ is connected.

Hence, $A=V(G) \cap D$ is ns-dominating in $G$.

Let $v \in V(G) \cap D$. Suppose $V\left(H^{v}\right) \backslash D \neq \emptyset$. Let $u \in V\left(H^{v}\right) \backslash D$. Since $V(G) \cap D \neq V(G)$, $\exists z \in V(G) \backslash D$. Since $\langle V(G \circ H) \backslash D\rangle$ is connected, there exists a $u-z$ path with vertices from $V(G \circ H) \backslash D$. However, any $u-z$ path must have $v$ as a vertex. This is not possible. Hence, $V\left(H^{v}\right) \backslash D=\emptyset$. In effect, $V\left(H^{v}\right) \subseteq D$.

Suppose $v \in V(G) \backslash D$. Let $x \in V\left(H^{v}\right) \backslash\left[V\left(H^{v}\right) \cap\right.$ $D] .=V\left(H^{v}\right) \backslash D$. This implies that $x \in V(G \circ H) \backslash$ $D$. Since $D$ is dominating in $G \circ H, \exists y \in D$ such that $d_{G \circ H}(x, y)=1$. Consequently, $y=v$ or $y \in V\left(H^{v}\right)$. Since $y \in D$ and $v \in V(G) \backslash D, y \neq v$. In effect, $y \in V\left(H^{v}\right)$. Now, $d_{G \circ H}(x, y)=1$ implies that $d_{H^{v}}(x, y)=1$. Hence, $V\left(H^{v}\right) \cap D$ is dominating in $H^{v}$.

Therefore, $($ iii $)$ holds.

For the converse, suppose $(i)$ holds. Let $x \in V(G \circ H) \backslash D$. Since $V(G \circ H) \backslash D \subseteq V\left(H^{v}\right), x \in V\left(H^{v}\right)$ and $V(G) \subseteq D$. Thus, $v \in D$ and $d_{G \circ H}(x, v)=1$. Hence, $D$ is dominating in $G \circ H$. Accordingly, $D$ is ns-dominating in $G \circ H$.

Suppose (ii) holds. Let $x \in V(G \circ H) \backslash D$. Suppose $x \in V(G)$. Since $V\left(H^{x}\right) \cap D$ is dominating in $H^{x}, V\left(H^{x}\right) \cap D \neq \emptyset$. Let $u \in V\left(H^{x}\right) \cap D$. Then $d_{G \circ H}(u, x)=1$ Suppose $x \notin V(G)$. Then $\exists z \in V(G)$ such that $x \in V\left(H^{z}\right)$. Since $V\left(H^{z}\right) \cap D$ is dominating in $H^{z}$ and $x \in V\left(H^{z}\right) \backslash D, \exists t \in V\left(H^{z}\right) \cap D$ such that $d_{H z}(x, t)=1$. Thus, $D$ is dominating in $G \circ H$. Since $D \subseteq$ 
$\cup_{v \in V(G)}\left(V\left(H^{v}\right), V(G) \cap D=\emptyset\right.$. Consequently, $V(G \circ H) \backslash D$ $=\left[\cup_{v \in V(G)}\left(V\left(H^{v}\right) \backslash D\right] \cup V(G)\right.$.

Let $p, q \in V(G \circ H) \backslash D, p \neq q$. If $p, q \in V\left(H^{v}\right) \backslash D$ for some $v \in V(G)$, then $[p, v, q]$ is a path with vertices in $V(G \circ H) \backslash D$. If $p, q \in V(G)$, then there is a $p-q$ path $P$ in $G$ since $G$ is connected. Note that $P$ is also a path in $V(G \circ H) \backslash D$. Suppose $p \in V(G)$ and $q \in V\left(H^{v}\right) \backslash D$ for some $v \in V(G)$. If $p=v$, then we can choose the path $[p, q]$ with vertices in $V(G \circ H) \backslash D$. Suppose $p \neq v$. Since $G$ is connected, there is a path $P_{1}=$ $\left[u_{1}, u_{2}, \ldots, u_{n}\right]$ such that $u_{i} \in V(G), \forall i=1,2, \ldots, n$ where $u_{1}=p$ and $u_{n}=v$. Thus the path $P_{2}=\left[u_{1}, u_{2}, \ldots, u_{n}, q\right]$ is a $p-q$ path with vertices in $V(G \circ H) \backslash D$. Suppose $p \in V\left(H^{v}\right) \backslash$ $D$ and $q \in V\left(H^{w}\right) \backslash D$ for some $v, w \in V(G)$. Since $G$ is connected, there is a path $\left[u_{1}, u_{2}, \ldots, u_{n}\right]$ with vertices in $V(G)$ such that $u_{1}=v$ and $u_{n}=w$. Then $\left[p, u_{1}, u_{2}, \ldots, u_{n}, w\right]$ is a path with vertices in $V(G \circ H) \backslash D$. Hence, $\langle V(G \circ H) \backslash D\rangle$ is connected. Accordingly, $D$ is ns-dominating in $G \circ H$.

Suppose (iii) holds. Let $x \in V(G \circ H) \backslash D$. Suppose $x \in V(G)$, i.e., $x \in V(G) \backslash D=V(G) \backslash(V(G) \cap D)$. Since $V(G) \cap D$ is dominating in $G, \exists y \in V(G) \cap D$ such that $d_{G}(x, t)=1$. It follows that $d_{G \circ H}(x, y)=1$. Suppose $x \in V\left(H^{v}\right)$ for some $v \in$ $V(G)$, i.e., $x \in V\left(H^{v}\right) \backslash D$. Note that $d_{G \circ H}(x, v)=1$. If $v \in$ $D$, then we are done. Suppose $v \notin D$, i.e., $v \in V(G) \backslash D$. In this case, $V\left(H^{v}\right) \cap D$ is dominating in $H^{v}$. That is $\exists w \in V\left(H^{v}\right) \cap$ $D$ such that $d_{H z}(x, w)=1$. It follows that $d_{G \circ H}(x, w)=1$. Hence, $D$ is dominating in $G \circ H$.

Let $x, y \in V(G \circ H) \backslash D$. Suppose $x, y \in V(G)$. Then $x, y \in$ $V(G) \backslash D$. Since $V(G) \cap D$ is ns-dominating in $G,\langle V(G) \backslash D\rangle$ is connected. In effect, there is a $x-y$ path $P$ with vertices in $V(G) \backslash D) \subseteq V(G \circ H) \backslash D$.

Suppose $x \in V(G)$ and $y \in V\left(H^{v}\right)$ for some $v \in V(G)$, i.e., $x \in V(G) \backslash D$ and $y \in V\left(H^{v}\right) \backslash D$. If $x=v$, then we can take the path $[x, y]$. Suppose $x \neq v$. If $v \in$ $V(G) \cap D$, then $V\left(H^{v}\right) \subseteq D$. This contradicts the fact that $V\left(H^{v}\right) \backslash D \neq \emptyset$. Thus, $v \in V(G) \backslash D$. Consequently, $V\left(H^{v}\right) \backslash D$ is dominating in $H^{v}$, i.e., $V\left(H^{v}\right) \cap D \neq \emptyset$. Let $x \in V\left(H^{v}\right) \cap D$. Since $x, v \in V(G) \backslash D$ and $\langle V(G) \backslash D\rangle$ is connected, there exists an $x-v$ path $\left[u_{1}, u_{2}, \ldots, u_{n}\right]$ with vertices in $V(G) \backslash D$ such that $u_{1}=x$ and $u_{n}=v$. Thus, $\left[u_{1}, u_{2}, \ldots, u_{n}, y\right]$ is an $x-y$ path with vertices in $V(G \circ H) \backslash D$. Suppose $x, y \in V\left(H^{v}\right), x \neq y$ for some $v \in V(G)$. Then $x, y \in V\left(H^{v}\right) \backslash D$. If $v \in D$, then $V\left(H^{v}\right) \subseteq D$. This contradicts the fact that $V\left(H^{v}\right) \backslash D \neq \emptyset$. Thus, $v \notin D$, i.e., $v \in V(G) \backslash D$. Now, $[x, v, y]$ is a path with vertices in $V(G \circ H) \backslash D$.

Suppose $x \in V\left(H^{v}\right)$ and $y \in V\left(H^{w}\right)$ for some $v, w \in V(G)$, $v \neq w$. Then $x \in V\left(H^{v}\right) \backslash D$ and $y \in V\left(H^{w}\right) \backslash D$. If $v \in D$ or $w \in D$, then $V\left(H^{v}\right) \subseteq D$ and $V\left(H^{w}\right) \subseteq D$. This is a contradiction since $V\left(H^{v}\right) \backslash D \neq \emptyset$ and $V\left(H^{w}\right) \backslash D \neq \emptyset$. Thus, $v, w \notin D$, i.e., $v, w \notin V(G) \backslash D \subseteq V(G \circ H) \backslash D$. Since, $\langle V(G) \backslash D\rangle$ is connected, there is a path $\left[u_{1}, u_{2}, \ldots, u_{n}\right]$ with vertices in $V(G) \backslash D$, and $u_{1}=v$ and $u_{n}=w$. Consequently, $\left[x, u_{1}, u_{2}, \ldots, u_{n}, y\right]$ is a path with vertices in $V(G \circ H) \backslash D$. Hence, $\langle V(G \circ H) \backslash D\rangle$ is connected. Accordingly, $D$ is nsdominating in $G \circ H$.

COROLlary 2.6. Let $G$ and $H$ be connected graphs. Then

$$
\gamma_{n s}(G \circ H)=\left\{\begin{array}{cl}
1 & \text { if }|G|=1 \\
|G| \cdot \gamma(H) & \text { otherwise. }
\end{array}\right.
$$

Proof. The case where $|G|=1$ follows from Corollary 2.2. Suppose $|G|=m \geq 2$. For each $v \in V(G)$, let $H^{v}$ be a copy of $H$ corresponding to vertex $v$. Further, for each $v \in V(G)$, let $D^{v}$ be a minimum dominating set in $H^{v}$. Then by Theorem 2.5 (ii), $D=\cup_{v \in V(G)} D^{v}$ is ns-dominating in $(G \circ H)$. Thus,

$$
\begin{aligned}
\gamma_{n s}(G \circ H) \leq|D| & =\left|\cup_{v \in V(G)} D^{v}\right|=\Sigma_{v \in V(G)}\left|D^{v}\right| \\
& =\Sigma_{v \in V(G)} \gamma(H)=|G| \cdot \gamma(H)
\end{aligned}
$$

Suppose $\exists D^{*}$ such that $D^{*}$ satisfies $(i)$ of Theorem 2.5. Then by Theorem 2.5, $D^{*}=\cup_{j \neq i} V\left(H^{v_{j}}\right) \cup\left\{V\left(H^{v_{i}}\right) \backslash D^{*}\right\}$. Now,

$$
\begin{aligned}
\left|D^{*}\right| & =\left|\cup_{j \neq i} V\left(H^{v_{j}}\right) \cup\left\{V\left(H^{v_{i}}\right) \backslash D^{*}\right\}\right| \\
& =\sum_{j \neq i}\left|H^{v_{j}}\right|+\left|V\left(H^{v_{i}}\right) \backslash D^{*}\right| \geq(m-1)|H|
\end{aligned}
$$

By Lemma 2.2, $\gamma(H) \leq \frac{|H|}{2}$, i.e., $2 \gamma(H) \leq|H|$. Hence,

$$
\begin{aligned}
\left|D^{*}\right| & \geq(m-1)(2 \gamma(H)+r)+t \\
& =(m-1) \gamma(H)+(m-1) \gamma(H) \\
& \geq(m-1) \gamma(H)+\gamma(H)=|G| \cdot \gamma(H) .
\end{aligned}
$$

Suppose $D^{*}$ satisfies $(i i)$ of Theorem 2.5. Then

$$
\begin{aligned}
\left|D^{*}\right| & =\sum_{v \in V(G} \mid V\left(H^{v} \cap D \mid \geq \sum_{v \in V(G)} \gamma\left(H^{v}\right)\right. \\
& =\sum_{v \in V(G} \gamma(H)=|G| \cdot \gamma(H) .
\end{aligned}
$$

Next, suppose $D^{*}$ satisfies (iii) of Theorem 2.5. Let $A=\left\{v_{1}, v_{2}, \ldots, v_{r}\right\}$, where $1 \leq r \leq m$. Then $D^{*}=$ $\bigcup_{v_{i} \in A} V\left(H^{v_{i}}\right) \bigcup_{v_{j} \in V(G) \backslash A}\left(D^{*} \cap \bar{V}\left(H^{v_{j}}\right)\right)$. Now

$$
\begin{aligned}
\left|D^{*}\right| & =r|H|+(m-r) \gamma(H) \geq r(2 \gamma(H))+(m-r) \gamma(H) \\
& =\gamma(H)(m+r)>m \gamma(H)=|D| .
\end{aligned}
$$

Therefore, $D$ is a minimum ns-dominating set in $G \circ H$, i.e., $\gamma_{n s}(G+H)=2$.

\section{INVERSE NON-SPLIT DOMINATION IN JOIN AND CORONA OF GRAPHS}

REMARK 3.1. For any graph $G, \gamma_{n s}^{\prime}(G) \geq 1$, if it exists.

THEOREM 3.2. Let $G$ and $H$ be connected graphs. Then

$$
\gamma(G+H)=\left\{\begin{array}{l}
1 \text { if } \operatorname{ext}(G) \neq \emptyset \text { or } \operatorname{ext}(H) \neq \emptyset \\
2 \text { if } \operatorname{ext}(G)=\emptyset \text { and } \operatorname{ext}(H)=\emptyset .
\end{array}\right.
$$

Proof. Suppose $\operatorname{ext}(G) \neq \emptyset$. Let $D=\{a\}$, where $a \in \operatorname{ext}(G)$ and $x \in V(G+H) \backslash D$. If $x \in V(G) \backslash D$, then $d_{G}(x, a)=1$ since $a \in \operatorname{ext}(G)$. It follows that $d_{G+H}(x, a)=$ 1. If $x \in V(H) \backslash D$, then $d_{G+H}(x, a)=1$. Thus, $D$ is dominating in $G+H$. Hence, $\gamma(G+H)=1$. The proof is similar for the $\operatorname{ext}(H) \neq \emptyset$.

Suppose now that Suppose $\operatorname{ext}(G)=\emptyset$ and $\operatorname{ext}(H)=\emptyset$. Let $D=\{a, b\}$, where $a \in V(G)$ and $b \in V(H)$. Let $x \in V(G+$ $H) \backslash D$. If $x \in V(G) \backslash D$. If $x \in V(G)$, then $d_{G+H}(x, b)=1$. If $x \in V(H), d_{G+H}(x, a)=1$. Thus, $D$ is dominating in $G+H$, implying that $\gamma(G+H) \leq 2$. Suppose $\gamma(G+H)=1$. Let $D=$ $\{t\}$ be dominating in $G+H$. If $t \in V(G)$, then $d_{G+H}(t, y)=1$ for all $y \in V(G)$, making $t$ an extreme vertex in $G$. This is a contradiction. The case where $t \in V(H)$ yields a similar result. Consequently, $\gamma(G+H) \neq 1$.

Accordingly, $\gamma(G+H)=2$.

THEOREM 3.3. Let $G$ be a nontrivial graph. Then $\gamma_{n s}^{\prime}\left(G+K_{1}\right)=\gamma(G)$.

Proof. By Theorem 3.2, $\gamma\left(G+K_{1}\right)=1$. Suppose $\operatorname{ext}(G)=\emptyset$. Let $D=\{a\}$ where $a \in V\left(K_{1}\right)$ and $D^{\prime}$ be a minimum dominating set in $G$. Then $D^{\prime} \subseteq V\left(G+K_{1}\right) \backslash D$. Moreover, $D^{\prime}$ is a dominating set in $G+K_{1}$ and $\left\langle V\left(G+K_{1}\right) \backslash D^{\prime}\right\rangle$ is connected. Thus, $D^{\prime}$ is ins-dominating set in $G+K_{1}$. Hence, $\gamma_{n s}^{\prime}\left(G+K_{1}\right) \leq\left|D^{\prime}\right| \gamma(G)$.

Suppose $D_{0}^{\prime}$ is an ins-dominating set in $G+K_{1}$ with respect to a dominating set $D_{0}$ in $G+K_{1}$. Let $D_{0}=\{t\}$. Suppose 
$t \in V(G)$. Then $d_{G+K_{1}}(t, x)=1 \forall x \in V(G)$. This implies that $t \in \operatorname{ext}(G)$, acontradiction. Thus, $t=a$. Since $D_{0}^{\prime}$ is dominating in $G+H$ and $D_{0}^{\prime} \subseteq V\left(G+K_{1}\right) \backslash D_{0}, D_{0}^{\prime}$ is dominating in $G$. Thus,

$$
\gamma_{n s}^{\prime}\left(G+K_{1}\right)=\left|D_{0}^{\prime}\right| \geq\left|D^{\prime}\right|=\gamma(G)
$$

Hence, $\gamma_{n s}^{\prime}\left(G+K_{1}\right)=\gamma(G)$.

THEOREM 3.4. Let $G$ and $H$ be nontrivial connected graphs. Then

$$
\gamma_{n s}^{\prime}(G+H)= \begin{cases}1 & \text { if }|\operatorname{ext}(G)|+|\operatorname{ext}(H)| \geq 2 \\ 2 & \text { if }|\operatorname{ext}(G)|+|\operatorname{ext}(H)|=0,1 .\end{cases}
$$

Proof. Suppose $|\operatorname{ext}(G)|+|\operatorname{ext}(H)|=0$, i.e., $|\operatorname{ext}(G)|=$ $\emptyset$ and $|\operatorname{ext}(H)|=\emptyset$. By Theorem 3.2, $\gamma(G+H)=2$. As shown in the Theorem, $D=\{a, b\}$ where $a \in V(G)$ and $b \in V(H)$, is a minimum dominating set in $G+H$. Since $|\operatorname{ext}(G)|=\emptyset$ and $|\operatorname{ext}(H)|=\emptyset,|V(G)| \geq 2$ and $|V(H)| \geq 2$. Let $D^{\prime}=$ $\left\{a^{\prime}, b^{\prime}\right\}$ where $a^{\prime} \in V(G) \backslash\{a\}$ and $b^{\prime} \in V(H) \backslash\{b\}$. That is $D^{\prime} \subseteq V(G) \backslash D$. Let $x \in V(G+H) \backslash D^{\prime}$. If $x \in V(G) \backslash D^{\prime}$, then $d_{G+H}\left(x, b^{\prime}\right)=1$. If $x \in V(H) \backslash D^{\prime}$, then $d_{G+H}\left(x, a^{\prime}\right)=1$. Thus, $D^{\prime}$ is dominating in $G+H$.

Let $u, v \in V(G+H) \backslash D^{\prime}$ with $u \neq v$. Let $P$ be defined as follows:

$$
P=\left\{\begin{array}{cl}
{[u, a, v]} & \text { if } u, v \in V(H) \backslash\left\{b^{\prime}\right\} \\
{[u, b, v]} & \text { if } u, v \in V(G) \backslash\left\{a^{\prime}\right\} \\
{[u, v]} & \text { if otherwise }
\end{array}\right.
$$

Thus, $\left\langle V(G+H) \backslash D^{\prime}\right\rangle$ is connected.

Hence, $D^{\prime}$ is ins-dominating set in $G+H$. Accordingly, $\gamma_{n s}^{\prime}(G+H) \leq 2$

Suppose $\gamma_{n s}^{\prime}(G+H)=1$. Then there is a dominating set $D_{1}$ and an ins-dominating set $D_{1}^{\prime}$ with respect to $D_{1}$ such that $\left|D_{1}\right|=2$ and $\left|D_{1}^{\prime}\right|=1$. Let $D_{1}^{\prime}=\{t\}$. If $t \in V(G)$, then $d_{G}(x, t)=$ $d_{G+H}(x, t)=1$ and so $t \in \operatorname{ext}(G)$, a contradiction. The same argument holds if $t \in \operatorname{ext}(H)$ Hence, $\gamma_{n s}^{\prime}(G+H)=2$.

Suppose $|\operatorname{ext}(G)|+|\operatorname{ext}(H)|=1$. Assume without loss of generality that $\operatorname{ext}(G)=\{a\}$ and $\operatorname{ext}(H)=\emptyset$. By Theorem 3.2, $\gamma(G+H)=1$. Clearly, $D=\{a\}$ is a minimum dominating set in $G+H$. Let $D^{\prime}=\{b, c\}$, where $b \in V(G) \backslash\{a\}$ and $c \in V(H)$, i.e., $D^{\prime} \subseteq V(G+H) \backslash D$. Now, $D^{\prime}$ is dominating in $G+H$.

Let $u, v \in V(G+H) \backslash D^{\prime}$ with $u \neq v$. Let $P$ be defined as follows:

$$
P=\left\{\begin{array}{cl}
{[u, a, v]} & \text { if } u, v \in V(H) \backslash\{c\} \\
{[u, c, v]} & \text { if } u, v \in V(G) \backslash\{b\} \\
{[u, v]} & \text { if otherwise }
\end{array}\right.
$$

Thus, $\left\langle V(G+H) \backslash D^{\prime}\right\rangle$ is connected.

Hence, $D^{\prime}$ is ins-dominating set in $G+H$. Accordingly, $\gamma_{n s}^{\prime}(G+H) \leq 2$.

Suppose $\gamma_{n s}^{\prime}(G+H)=1$. Let $D_{0}^{\prime}=\{t\}$ be an ins-dominating set in $G+H$ with respect to some dominating set $D_{0}$. Since $D_{0}^{\prime}$ is dominating in $G+H, d_{G}(t, x)=1 \forall x \in V(G)$. It follows that $t \in \operatorname{ext}(G)$, so $t=a$. Let $D_{0}=\{p\}$. Since, $D_{0}$ is dominating in $G+H, p \in \operatorname{ext}(G) \cup \operatorname{ext}(H)=$ $\operatorname{ext}(G)$. Thus, $p=a$. This contradicts the fact that $D_{0}^{\prime} \subseteq V(G+H) \backslash D_{0}$. Hence, $\gamma_{n s}^{\prime}(G+H)=2$.

Suppose $|\operatorname{ext}(G)|+|\operatorname{ext}(H)| \geq 2$. By Theorem 3.2, $\gamma(G+H)=1$. Consider the following cases:

Case 1. $\operatorname{ext}(G)=\emptyset$. Then $|\operatorname{ext}(H)| \geq 2$. Let $a, b \in \operatorname{ext}(G)$, $a \neq b$. Following the proof of Theorem, $D=\{a\}$ is a minimum dominating set in $G+H$. Let $D^{\prime}=\{b\}$. Clearly, $D^{\prime} \subseteq V(G+H) \backslash D$. Moreover, $D^{\prime}$ is a dominating set in $G+H$. Furthermore, $\left\langle V(G+H) \backslash D^{\prime}\right\rangle$ is connected. Thus, $D^{\prime}$ is an ins-dominating set in $G+H$ with respect to $D$. Hence, $\gamma_{n s}^{\prime}(G+H) \leq 1$. By Lemma, $\gamma_{n s}^{\prime}(G+H)=1$.

Case 2. $\operatorname{ext}(\bar{H})=\emptyset$. Proof of this is similar to Case 1 .

Case 3. $\operatorname{ext}(G) \neq \emptyset$ and $\operatorname{ext}(H) \neq \emptyset$. Let $D=\{a\}$, where $a \in \operatorname{ext}(G)$. Then $D$ is a dominating set in $G+H$. Let $D^{\prime}=$ $\{b\}$, where $b \in \operatorname{ext}(H)$. Clearly, $D^{\prime} \subseteq V(G+H) \backslash D$ and $D^{\prime}$ is dominating in $G+H$. Moreover, $\left\langle V(G+H) \backslash D^{\prime}\right\rangle$ is connected. Thus, $D^{\prime}$ is an ins-dominating set in $G+H$ with respect to $D$. Hence, $\gamma_{n s}^{\prime}(G+H) \leq 1$. Accordingly, $\gamma_{n s}^{\prime}(G+$ $H)=1$.

The next two results will be useful in the succeeding theorems.

THEOREM 3.5. [4] Let $G$ be a connected graph of order $m$ and let $H$ be any graph of order $n$. Then $C \cap V(G \circ H)$ is a dominating set in $G \circ H$ if and only if $V\left(v+H^{v}\right) \cap C$ is a dominating set of $v+H^{v}$ for every $v \in V(G)$.

COROLlary 3.6. [4] . Let $G$ be a connected graph of order $m$ and let $H$ be any graph of order $n$. Then $\gamma(G \circ H)=m$.

LEMMA 3.7. Let $G$ and $H$ be connected graphs and $D$ be a minimum dominating set in $G \circ H$. If $\gamma(H) \geq 2$, then $D=$ $V(G)$.

Proof. Suppose $\gamma(H) \geq 2$. By Theorem 3.5, $D \cap(V(a+$ $\left.H^{a}\right)$ ) is dominating in $H^{a}, \forall a \in V(G)$. Suppose $\exists t \in V(G)$ such that $\mid D \cap\left(V\left(t+H^{t}\right) \mid \geq 2\right.$. Then by Corollary 3.6,

$$
\begin{aligned}
|G| & =|D|=\sum_{a \in V(G)}\left|D \cap V\left(a+H^{a}\right)\right| \\
& =\sum_{a \neq t}\left|D \cap V\left(a+H^{a}\right)\right|+\left|D \cap V\left(t+H^{t}\right)\right| \\
& \geq \sum_{a \neq t} 1+2=(|G|-1)+2=|G|+1 .
\end{aligned}
$$

This is a contradiction. Thus, $\mid D \cap\left(V\left(a+H^{a}\right) \mid=1\right.$. Suppose $\exists a \in V(G)$ such that $\mid D \cap\left(V\left(H^{a}\right) \mid \neq \emptyset\right.$. Then $\mid D \cap\left(V\left(H^{a}\right) \mid=1\right.$. Since $D$ is dominating in $G \circ H$ and $a \in V(G), D \cap\left(V\left(H^{a}\right)\right.$ is dominating in $H^{a}$. Consequently,

$$
\gamma(H)=\gamma\left(H^{a}\right) \leq \mid D \cap\left(V\left(H^{a}\right) \mid=1\right.
$$

This is a contradiction. Hence, $D \cap\left(V\left(H^{a}\right)=\emptyset\right.$, $\forall a \in V(G)$. Accordingly, $D \subseteq V(G)$. Since, $D \cap(V(a+$ $\left.H^{a}\right) \neq \emptyset, \forall a \in V(G), a \in D, \forall a \in V(G)$. That is, $V(G) \subseteq D$. Therefore, $D=V(G)$.

LEMMA 3.8. Let $G$ and $H$ be connected graphs such that $\gamma(H)=1$. Then $D$ is a minimum dominating set in $G \circ H$ if and only if one of the following conditions hold:

(i) $D=V(G)$;

(ii) $D=(V(G) \backslash A) \cup\left\{t_{a}: a \in A\right\}$ for some $A \subseteq V(G)$ and $t_{a} \in \operatorname{ext}\left(H^{a}\right)$

Proof. If $D=V(G)$, then we are done. Suppose $D \neq$ $V(G)$. Let $A=V(G) \backslash D$. Since $D$ is dominating in $G \circ H$, $V\left(H^{a}\right) \cap D$ is dominating in $H^{a} \forall a \in A$. Now, $\gamma(H)=1$ so $\left|V\left(H^{a}\right) \cap D\right|=1$. It follows that $V\left(H^{a}\right) \cap D=\left\{t_{a}\right\}, \forall a \in A$. Thus, $D=(V(G) \backslash A) \cup\left\{t_{a}: a \in A\right\}$.

Conversely, if $D=V(G)$, then $D$ is a min dominating set in $G \circ H$. Suppose (ii) holds. Then

$$
|D|=|V(G) \backslash A|+|A|=|G|
$$


Let $x \in V(G \circ H) \backslash D$. If $x \in V\left(H^{a}\right)$ for some $a \in V(G) \backslash A$, then $d_{G \circ H}(a, x)=1$. If $x \in A$, then $d_{G \circ H}\left(x, t_{x}\right)=1$. Thus, $D$ is a minimum dominating set in $G \circ H$.

THEOREM 3.9. Let $G$ and $H$ be connected graphs. Then

$$
\gamma_{n s}^{\prime}(G \circ H)=|G| \gamma(H)
$$

Proof. Let $D=V(G)$. Then $D$ is a minimum dominating set in $G \circ H$. For each $a \in V(G)$, Let $D_{a}$ be a minimum dominating set in $H^{a}$. Let $D^{\prime}=\cup_{a \in V(G)} D_{a}$. Then $D^{\prime} \subseteq V(G \circ H) \backslash \subseteq D$. By Lemma 3.8, $D^{\prime}$ is dominating in $G \circ \bar{H}$.

Let $u, v \in V\left(G \circ H \backslash D^{\prime}\right.$. If $u, v \in V\left(H^{a}\right) \backslash D_{a}$ for some $a \in V(G)$, then $[u, a, v]$ is a path with vertices in $V(G \circ H)$ $D$. If $u \in V(G)$ and $v \in V\left(H^{u}\right) \backslash D_{u}$, then we consider the path $[u, v]$. If If $u, v \in V(G)$, then there is a path with vertices in $V(G \circ H) \backslash D$ since $G$ is connected. If If $u \in V(G)$ and If $v \in V\left(H^{w}\right)$ for some $w \in V(G)$, then consider the path $\left[u_{1}, u_{2}, \ldots, u, v\right]$, where $u_{1}=u, u_{n}=w$ and $\left[u_{1}, u_{2}, \ldots, u_{n}\right]$ is a $u-v$ path in $G$. If $u \in V\left(H^{a}\right) \backslash D_{a}$ and $v \in V\left(H^{b}\right) \backslash D_{b}$, then consider the path $\left[u_{1}, u_{2}, \ldots, u_{n}, v\right]$ where $u_{1}=u, u_{2}=$ $a, u_{n}=b$ and $\left[u_{2}, u_{3}, \ldots, u_{n}\right]$ is an $a-b$ path in $G$. Thus, $\left\langle V(G \circ H) \backslash D^{\prime}\right\rangle$ is connected.

Hence, $D^{\prime}$ is an ins-dominating set in $G \circ H$. Accordingly,

$$
\begin{aligned}
\gamma_{n s}^{\prime}(G \circ H) & \leq\left|D^{\prime}\right|=\left|\cup_{a \in V(G)} D_{a}\right| \\
& =\sum_{a \in V(G)}\left|D_{a}\right| \\
& =\sum_{a \in V(G)} \gamma(H) \\
& =|G| \gamma(H) .
\end{aligned}
$$

Let $D$ be a minimum dominating set in $G \circ H$ and $D^{\prime}$ be a minimum dominating set in $(G \circ H)$ and $D^{\prime}$ be a minimum insdominating set in $(G \circ H)$ with respect $D$. Suppose $\gamma(H) \geq 2$. Then by Lemma 3.7,D $=V(G)$. Since $D^{\prime}$ is dominating in $G \circ H$ and $D^{\prime} \subseteq V(G \circ H) \backslash D . D^{\prime} \cap V\left(H^{a}\right)$ is dominating in $H^{a}, \forall a \in V(G)$. Thus,

$$
\begin{aligned}
\gamma_{n s}^{\prime}(G \circ H) & =\left|D^{\prime}\right|=\left|\cup_{a \in V(G)}\left(D^{\prime} \cap V\left(H^{a}\right)\right)\right| \\
& \geq \sum_{a \in V(G)} \gamma\left(H^{a}\right)=\sum_{a \in V(G)} \gamma(H)=|G| \gamma(H) .
\end{aligned}
$$

Thus,

$$
\begin{aligned}
\gamma_{n s}^{\prime}(G \circ H) & =\mid \cup_{a \in V(G)}\left(D ^ { \prime } \cap \left(V\left(H^{a}\right) \cup\{a\} \mid\right.\right. \\
& =\sum_{a \in V(G)} \mid D^{\prime} \cap\left(V\left(H^{a}\right) \cup\{a\} \mid\right. \\
& \geq \sum_{a \in V(G)} 1=|G|=|G| \gamma(H) .
\end{aligned}
$$

Suppose $\gamma(H)=1$. Since $D^{\prime}$ is dominating in $G \circ H$, $D^{\prime} \cap\left(V\left(H^{a}\right) \cup\{a\} \neq \emptyset\right.$. Thus, $\gamma_{n s}^{\prime}(G \circ H)=|G| \gamma(H)$.

\section{CONCLUSION}

The last few decades have seen how graph theory were useful in the area of computer science, particularly in developing algorithms and in computer communications network. In [2], Balasundaram mentioned the role of dominating sets in clustering problem in ad hoc network topologies. An important use of dominating sets and its inverse in information retreival system was cited in [1]. The author stated that the presence of a secondary set of nodes to pass on the information is essential since it serves as back up in case the primary set of nodes fails. The concept of inverse non-split dominating set was first introduced by Bibi, et. al. in the same paper. Interesting results on inverse nonsplit domination number on special classes of graphs were given . Relationships with associated domination invariants were also investigated. This present work invetigated intensively the abovementioned parameters in some graphs resulting from binary operations, such as the join and corona of graphs, which were not tackled by Bibi, et. al. Important results on characterization of non-split and inverse non-split dominating sets were presented here and corresponding formulas for computing the non-split and inverse non-split dominating numbers were also explicitly given.

\section{REFERENCES}

[1] B. Balasundaram, S. Butenko. Graph domination, coloring and cliques in telecommunications. Handbook of Optimization in Telecommunications, pages 865-890. Springer, 2006.

[2] K. Ameenal Bibi., K. Selvakumar. The inverse split and nonsplit domination in graphs. International Journal of Computer Applications, (0975 -8887), No. 7, Vol 8, 2010.

[3] G.J. Chang. Algorithm Aspects of Domination in Graphs.

[4] C.E. Go, S.R. Canoy, Jr. Domination in the corona and join of graphs. International Mathematical Forum, Vol. 6, 2011, no. 16,763 - 771 .

[5] F. Harary, Graph Theory. Addison-Wesley, Reading MA (1969).

[6] V.R. Kulli, B. Janakiram. The split domination number of a graph. Graph Theory Notes of New York. New York Academy of Sciences. XXXII, pp.16-19.

[7] V.R. Kulli, B. Janakiram. The non-split domination number of a graph. The Journal of Pure and Applied Math, 31(5), pp.545-550, 2000.

[8] O. Ore. Theory of graphs. American Mathematical Society Colloquium Publications, Vol XXXVIII, American Mathematical Society, Providence, R.I. 1962. 